\title{
Photochemical Polymerization of Thiophene Derivatives in Aqueous Solution
}

Sergey A. Piletsky, Elena V. Piletska, Kal Karim, Frank Davis, Seamus P. J. Higson and Anthony P. F. Turner

Institute of BioScience and Technology, Cranfield University at Silsoe, Bedfordshire, $M K 45$ 4DT, $U K$.

E-mail: S.Piletsky@cranfield.ac.uk

Polythiophenes have been a subject of intensive research during the last two decades. These materials, containing a $\pi$-conjugated carbon backbone, exhibit interesting electrochemical, electrochromic and shielding properties, which provide a basis for various new technologies. ${ }^{1-3}$ Thiophene derivatives can be oxidized chemically ${ }^{2,4,5}$, photochemically $^{3,6}$ or electrochemically ${ }^{1}$ and polymerized to the corresponding oligo- or polythiophenes. However, the processability of those materials is rather poor which impedes their practical application. In particular, their solubility in most organic solvents and water is too low. An intensive research effort has been initiated aimed at the development of highly soluble and easily processible polythiophenes including, for example, alkylsulfanyl groups ${ }^{4}$ to render the polymer soluble in organic solvents or carboxylic $^{2}$ or sulphonic ${ }^{5}$ acids to confer water solubility. Despite the progress achieved in these studies, the yield of polythiophenes is often low and the process itself can be expensive and demand the use of aggressive and toxic solvents. 
We report here on the photopolymerization of some substituted thiophenes, 3thiophene acetic acid (TAA), boronic acid (TBA) and carboxylic acid (TCA), catalyzed by potassium dichromate. This reaction can be performed under mild conditions in an aqueous environment and using irradiation with visible light. The process leads to a high yield of oligomers (4-7mers), which are soluble in THF and the simultaneous deposition of insoluble polymer on the illuminated surface. Previous work within this department has shown the suitability of this process for making molecularly imprinted films for use in assays. ${ }^{7}$ A typical polymerization, that of TCA was performed as follows: $10 \mathrm{mmol}$ of 3-thiophenecarboxylic acid were dissolved in $50 \mathrm{ml}$ of deionized water and mixed with $50 \mathrm{ml}$ of $30 \mathrm{mmol}$ aqueous potassium dichromate. The solution was exposed to visible light $(100 \mathrm{~W})$ for 3 days. During the steady state irradiation with visible light, the solution became brown in colour, forming a dark brown precipitate and coating on the illuminated container surface. This was not observed when reactions were carried out in the dark, or when potassium dichromate was replaced by copper(II) chloride, ferric chloride, potassium permanganate or ammonium persulphate. The resulting suspension was evaporated and the brown residue washed extensively with water to remove nonreacted monomer and potassium dichromate. After drying the oligomers were extracted with refluxing THF. The yield of the oligomeric derivatives was $85 \%$. The same reaction was performed in polystyrene cuvettes $\left(10 \times 10 \times 45 \mathrm{~mm}^{3}\right.$, Sarstedt, Germany) which were used for measuring the UV-vis absorbance of the deposited film or in polycarbonate Petri dishes. Similar behaviour was found when TAA or TBA were used as the monomers.

Elemental analysis of the soluble fraction of polythiophene carboxylic acid gave: $\mathrm{C}-37.7 \% ; \mathrm{H}-2.8 \% ; \mathrm{S}-16.4 \% ; \mathrm{Cr}-12.8 \% ; \mathrm{K}-0.5 \%$. The lower value of $\mathrm{S} / \mathrm{C}$ ratio 
(0.44) compared to theoretical one $(0.53)$ is due to bond breaking of thiophene rings. ${ }^{6}$ Unfortunately it proved impossible to remove the chromium contaminant $\left(\mathrm{Cr}_{2} \mathrm{O}_{3}\right)$. Various methods were attempted, but only the most drastic such as hot alkaline persulphate solution removed Cr. However, this also appeared to affect the polymer composition, probably due to further oxidation.

GPC analysis of the product soluble in THF indicated formation of oligomers and polymer, the largest fraction (78\%) having molecular weight $0.6 \times 10^{3}$ (vs. PST), corresponding to the $4-5$ mer, with $20 \%$ having molecular weight $0.8 \times 10^{3}$ (vs. PST), corresponding to the 6-7mer, but with small peaks indicating higher molecular weight species of up to $2.5 \times 10^{3}$ (vs. PST). The results are similar to those for polymers obtained from UV light induced photodeposition performed using an excimer laser. ${ }^{6}$

Mass spectrometry measured of the soluble polymer gave peaks at 90,$155 ; 242$; $289 ; 421 ; 460 ; 613 ; 766 ; 863 ; 919 ; 1073 ; 1190 ; 1340 ; 1425 ; 1495 ; 1574 ; 2129$; 2518. MALDI experiments performed on the insoluble polymer showed masses peaking at about 1000 for PTCA and 1500 for PTBA. However MALDI may only be removing lower molecular weight fragments from the polymer mixture. The peaks were regularly spaced at intervals of 44 , indicating that either carboxylate or boronic sidegroups were being removed under the conditions used to obtain the spectra.

FT-IR measurements of the product showed adsorption bands specific to $\alpha$ coupled polythiophene. IR (cm-1): 3125, 1705, 1610, 1570, 1530, 1440, 1360, 940, 880, $840,760,710,670,630,540$. Broad peak between $3200-3700 \mathrm{~cm}^{-1}$ is characteristic for $\mathrm{HO}$ absorption of carboxylic function and the peak at 1075 characteristic of a carbonyl stretch. Those results clearly confirm that the carboxylic function withstood the applied 
oxidation conditions and remains in the resulting polymer. IR spectra of the monomers showed sharp peaks at about 3150 (TCA) and 3480 (TBA) corresponding to the $\mathrm{C}-\mathrm{H}$ stretch, these were much reduced in the polymeric product, indicating loss of those bonds due to polymerization.

The UV-vis spectrum (Fig. 1) of PTCA in DMF solutions displays adsorption maxima at 425 and $585 \mathrm{~nm}$, indicating that the polymer probably exists as mixture of two forms with different level of conjugation. ${ }^{4}$ The UV spectrum of the solid form deposited on the inside of a polystyrene cuvette showed a different spectrum with higher adsorption at longer wavelengths.

Ellipsometry studies and fitting of the data obtained showed that the PTCA film used for the UV measurements had a thickness of $0.59 \mu \mathrm{m}$ with a roughness of $6.4 \mathrm{~nm}$. The film had a refractive index of 1.63 at $800 \mathrm{~nm}$, increasing gradually to 1.65 at $500 \mathrm{~nm}$, 1.68 at $400 \mathrm{~nm}$ and 1.85 at $300 \mathrm{~nm}$.

AFM and SEM studies were performed on films deposited onto polystyrene and show flat films with some granular structure (Fig 2a). In an attempt to determine typical thicknesses, a film of PTCA was photodeposited onto a polycarbonate surface, the substrate was broken and analyzed using SEM; these images are shown in Figure 2. The film shows a cracked appearance and the cross-section pictures (Fig. 2b) give a thickness of 1-2 $\mu \mathrm{m}$. Energy Dispersive Spectrography (EDS) analysis gave a surface composition indicating lower levels of $\mathrm{Cr}$ composition as compared to the soluble fraction $(\mathrm{S}: \mathrm{Cr}$ atomic ratio about $4: 1)$.

A possible mechanism of the reaction is as follows. Due to the nucleophilic character of the - $\mathrm{COOH}$ group, 3-thiophenecarboxylic acid can not be polymerized using 
a traditional approach such as when $\mathrm{FeCl}_{3}$ is used as oxidizing agent. In some cases polymerization of acidic derivatives was performed using a two step procedure involving first protection of the acid with benzyl group. ${ }^{1}$ For the polymerization reaction to occur, three things must be present: monomer, dichromate ion and light. No reaction was observed when monomer and dichromate solutions were combined and stored in the dark and the reaction was not seen to occur when potassium dichromate was replaced by other oxidants. Dichromate ion has been shown to be catalytically active in a series of photochemical reactions. A complex of $\mathrm{Cr}(\mathrm{VI})-\mathrm{TCA}_{3}$ was shown to catalyse the photopolymerization $(440 \mathrm{~nm})$ of methyl methacrylate in $\mathrm{DMF}^{8}$. No presence of complexation between $\mathrm{Cr}_{2} \mathrm{O}_{7}{ }^{2-}$ and TCA however can be detected spectrophotometrically in the absence of illumination, which is in agreement with previous finding. ${ }^{9}$ According to the suggested mechanism of photochemical polymerization, the complex between monomer and $\mathrm{Cr}(\mathrm{VI})$, formed by photocomplexation undergoes a charge-transfer reaction leading after a charge separation to $\mathrm{Cr}(\mathrm{V})$ and an organic radical. ${ }^{9} \mathrm{Cr}(\mathrm{V})$ oxidizes the starting material, by a spin-pairing of two radical cations to form a dihydro-dimer dication, which subsequently undergoes a loss of protons and rearomatization ${ }^{8,10}$. Coupling occurs at the position of highest unpaired-electron spin density. The polymerization continues with the oxidation of the dimer which undergoes further coupling reaction with other radical cations. The final photoproducts are $\mathrm{Cr}_{2} \mathrm{O}_{3}$ and polyTCA.

In conclusion, we have demonstrated a novel polymerization method for $\alpha$-linked oligo- and polythiophenes. The present photochemical polymerization method provides a 
basis for coating a surface with layers of new functional materials $0.5-2 \mu \mathrm{m}$ thick and is potentially very useful for use in microelectronics and sensors.

\section{Acknowledgements}

The authors would like to acknowledge Prof. C. Stirling (Sheffield University) for help with the MALDI work, Colin Matthews (Cranfield University) for help with SEM, David Sheppard of Jobin Yvon Ltd for ellipsometry measurements and BBSRC for funding for FD as part of the Centre for Bioarray innovation within the post-genomic consortium. SP would like to acknowledge with gratitude receiving Royal Society-Wolfson Research Merit Award.

\section{References}

1. Li, G. T.; Kossmehl, G.; Welzel, H. P.; Engelmann, G.; Hunnius, W. D.; Plieth, W.; Zhu, H. S. Macromol. Chem. Phys. 1998, 199, 525-533.

2. Englebienne, P.; Weiland, M. Chem. Commun. 1996, 14, 1651-1652.

3. Fujitsuka, M.; Sato, T.; Segawa, H.; Shimidzu, T. Synth. Metals 1995, 69, 309-310.

4. Iarossi, D.; Mucci, A.; Schenetti, L.; Seeber, R.; Goldoni, F.; Affronte, M.; Nava, F. Macromolecules, 1999, 32, 1390-1397.

5. Chayer, M.; Faid, K.; Leclerc, M. Chem. Mater. 1997, 9, 2902-2905.

6. Nishio, S.; Matsuzaki,_A.; Sato, H. Synth. Met. 1997, 184, 367-368.

7. Piletsky, S. A.; Piletska, E. V.; Bossi, A.; Karim, K.; Lowe, P.; Turner A. P. F. Biosens. Bioelec. 2001, 16, 707.

8. Chaturvedi, B.; Srivastava, A. K. J. Photochem. Photobiol.-Chem. 1992, 64, 183-189. 
9. Fageol, P.; Bolte, M.; Lemaire, J. J. Phys. Chem. 1988, 92, 239-243.

10. Frechette, M.; Belletete, M.; Bergeron, J.-Y.; Durocher, G.; Leclerc, M. Synth. Met. 1997, 84, 223-224.

\section{List of Figures}

Figure 1. Absorbance spectra of the PTCA and TCA solutions and films. Suspension of $10 \mathrm{mg} / \mathrm{ml} \mathrm{PTCA}$ in DMF was stirred for $20 \mathrm{~min}$ at $80 \mathrm{C}$, filtered through the microfilter from the solid and its absorbance measured spectrophotometrically. A $1 \mathrm{mg} / \mathrm{ml}$ solution of TCA in DMF was used. PTCA was photodeposited as a solid film inside a polystyrene cuvette.

Figure 2 (a) AFM of a photodeposited film of PTBA (b) SEM crossection of a photodeposited film of PTCA. 


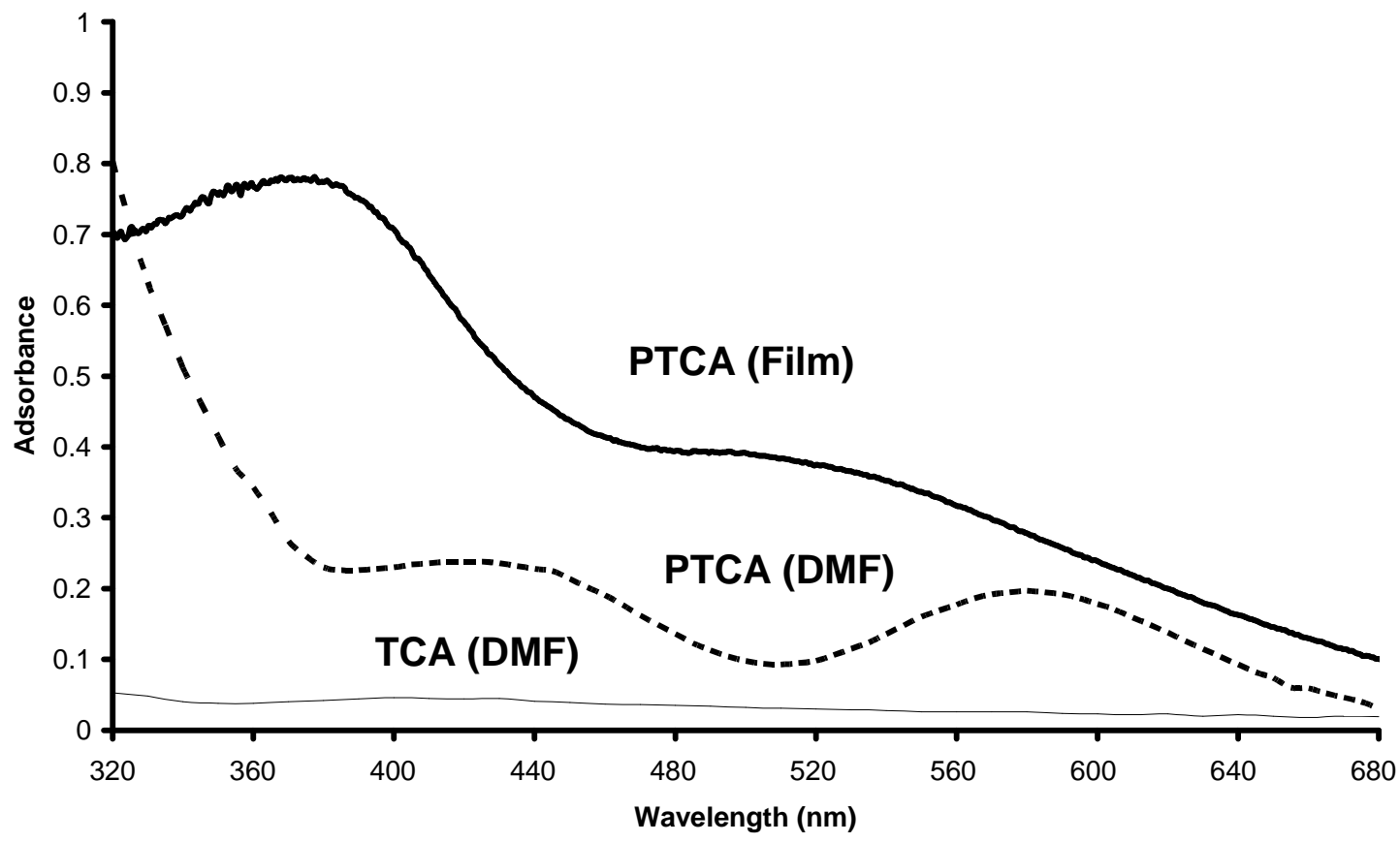




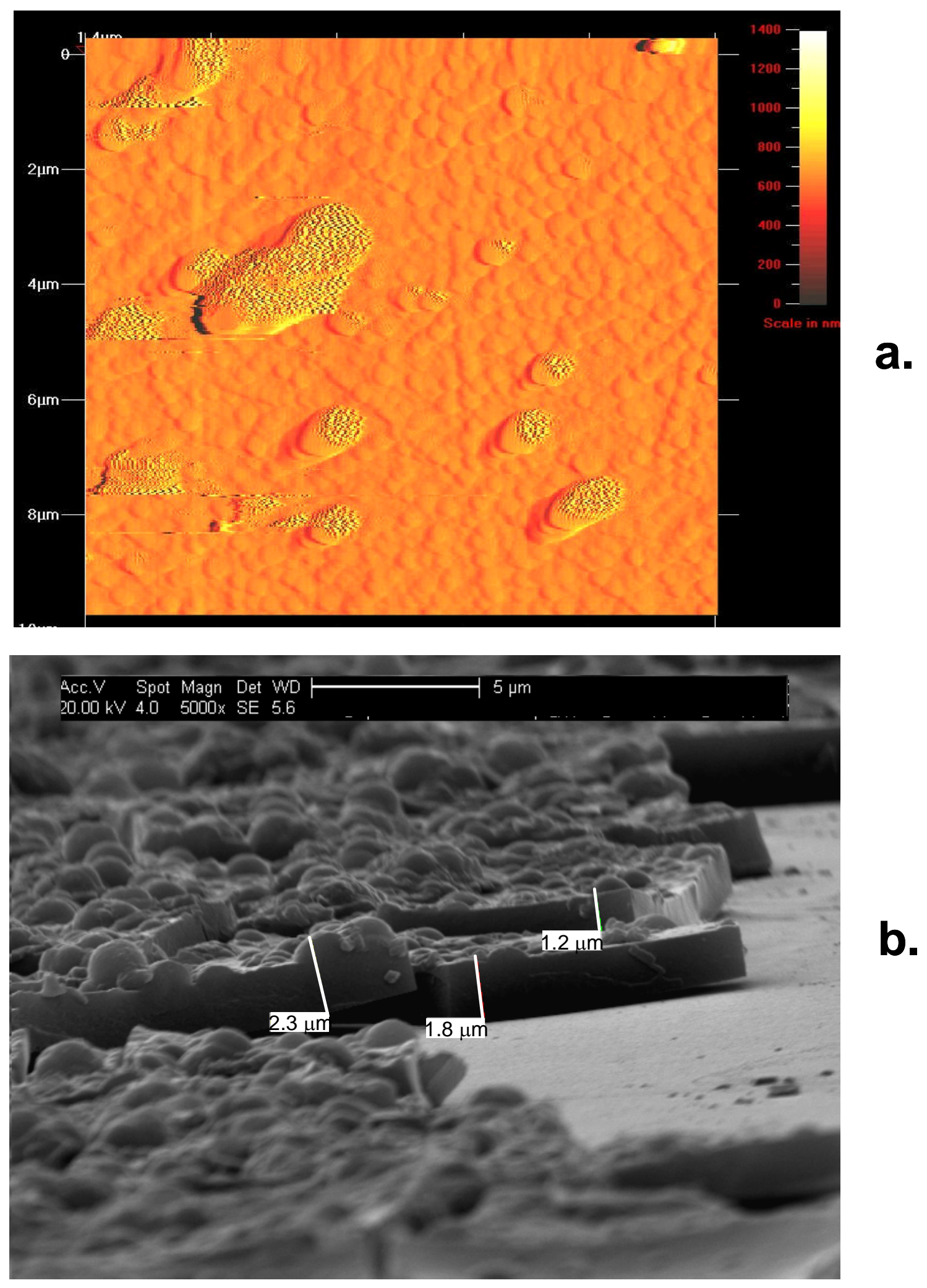


Potassium dichromate, 3-thiophenecarboxylic (TCA), 3-thiophenecacetic (TAA) and 3thiopheneboronic acid (TBA) were purchased from Aldrich. All reagents were used as received.

UV-vis spectra were recorded on a Shimadzu UV2100 spectrophotometer. For the IR spectra a Bruker Equinox55 FT-IR spectrometer was used and the samples were dispersed in $\mathrm{KBr}$ discs. Gel permeation chromatography (GPC) was carried out using a FPLC system (Pharmacia, Sweden) equipped with a UV detector, using DMF as eluent. The average molecular weights were calculated using a calibration curve of monodisperse polystyrene standards.

Mass spectrometry on the soluble fractions were carried out using VG ZAB 2SE double focusing magnetic sector instrument capable of high mass range (15000 mass units) and high resolution work. MALDI spectra were recorded using a Bruker Reflex III mass spectrometer and using a dihydroxybenzoic acid matrix.

SEM micrographs were obtained using a Oxford Instruments FEI XL30 SFEG. Ellipsometry was performed on a sample deposited inside a polystyrene cuvette using a UVISEL variable angle scanning instrument with a spot size of $0.5 \mathrm{~mm}$ and an integration time of $0.2 \mathrm{~s}$. Atomic force microscope (AFM) images were obtained using the Quesant Resolver model (Quesant Instrument Corporation, Hills, CA). Samples were scanned in wave-mode, at a scan rate of $2 \mathrm{~Hz}$ and 400-pixel resolution. 